\title{
Multi-Input Square Iterative Learning Control with Bounded Inputs*
}

\author{
Brian J. Driessen \\ Structural Dynamics Department \\ Sandia National Laboratories \\ Albuquerque, NM 87185-0847 \\ bjdries@sandia.gov \\ Nader Sadegh \\ Department of Mechanical Engineering \\ Georgia Institute of Technology \\ Atlanta, GA 30332 \\ nader.sadegh@me.gatech.edu \\ Kwan S. Kwok \\ Robotics Center \\ Sandia National Laboratories \\ Albuquerque, NM 87185-1003 \\ kskwok@sandia.gov
}

\begin{abstract}
In this paper we present a very simple modification of the iterative learning control algorithm of Arimoto et al [1] to the case where the inputs are bounded. The Jacobian condition presented in Avrachenkov [2] is specified instead of the usual condition specified by Arimoto et al [1]. (See also Moore [11].) In particular, the former is a condition for monotonicity in the distance to the solution instead of monotonicity in the output error. This observation allows for a simple extension of the methods of Arimoto et al [1] to the case of bounded inputs since the process of moving an input back to a bound if it exceeds it does not affect the contraction mapping property; in fact, the distance to the solution, if anything, can only decrease even further. The usual Jacobian error condition, on the other hand, is not sufficient to guarantee the chopping rule will converge to the solution, as proved herein. To the best of our knowledge, these facts have not been previously pointed out in the iterative learning control literature.
\end{abstract}

\section{Introduction}

Learning control is a method of control that feeds the system inputs for a specific task repetitively and uses the actual on-line measured response of the system to evaluate the quality or goodness of the input. The actual responses are used in a feedback loop in which the inputs are adjusted to reduce measured errors in the output. Example applications include robotics and manufacturing where a certain output tracking task is to be performed repeatedly. Usually the output is the position or velocity history of the robot's joints although sometimes it also includes measured forces at the end effector (see Cheah and Wang [4]).

Learning control has a history dating back to 1984 (see Arimoto et al [1]) when it was first applied to robot motion control. Horowitz [10] gives a nice history of the development and usage of learning controllers for (rigid) robot manipulators. He compares and contrasts different learning algorithms and also provides an experimental demonstration of a robot that learns to make its end effector track a circular trajectory. He insightfully points out that an open area of research is in finding methods for robust optimal (e.g., minimum energy, minimum vibration, or minimum time) trajectory learning, as opposed to only finding a control history that meets output requirements. Examples of work that have empirically investigated approaches to this problem include Gorinevsky ([7], [8], and [9]), who considered the use of the LevenbergMarquardt optimization method for least squares, and

\footnotetext{
* Sandia is a multiprogram laboratory operated by Sandia Corporation, a Lockheed Martin Company, for the United States Department of Energy under Contract DE-AC04-94AL85000.
} 


\section{DISCLAIMER}

This report was prepared as an account of work sponsored by an agency of the United States Government. Neither the United States Government nor any agency thereof, nor any of their employees, make any warranty, express or implied, or assumes any legal liability or responsibility for the accuracy, completeness, or usefulness of any information, apparatus, product, or process disciosed, or represents that its use would not infringe privately owned rights. Reference herein to any specific commercial product, process, or service by trade name, trademark, manufacturer, or otherwise does not necessarily constitute or imply its endorsement, recommendation, or favoring by the United States Government or any agency thereof. The views and opinions of authors expressed herein do not necessarily state or reflect those of the United States Government or any agency thereof. 


\section{DISCLAIMER}

Portions of this document may be illegible in electronic image products. Images are produced from the best available original document. 
Sadegh and Driessen [12] who considered the use of gradient-based algorithms for constrained optimization.

Cheng and Peng [5] consider learning control with input bounds and modeling error. However, the methodology and convergence theory was restricted to single-input/single-output systems.

The present paper utilizes a key result in Avrachenkov [2] to extend the existing learning control approach initiated by Arimoto et al [1] to the case where the inputs are bounded. If $J$ is the true Jacobian and $\hat{J}$ the approximate model-based Jacobian, the condition for the method of Arimoto et al [1] is one associated with monotonicity of the sequence of output error norms and is given by $\left\|I-J \hat{J}^{-1}\right\|<1$. Simply switching the $J$ and $\hat{J}^{-1}$ in this norm condition gives us a condition for monotonicity of the sequence of input error norms, i.e., $\left\|I-\hat{J}^{-1} J\right\|<1$ (see Avrachenkov [2]). Under this latter norm condition, an iterative learning control scheme analogous to an inputbounded version of the method initiated by Arimoto et al [1] results. The associated method, theorem, and proof will be given in section 2 and conclusions in section 3 .

\section{Bounded Multi-Input Iterative Learning Control}

Let us review briefly the method of Arimoto et al [1]. We have the following continuous time system:

$$
\begin{aligned}
& \dot{x}=\hat{f}(x)+\hat{g}(x) u \\
& y=C x+d(t)
\end{aligned}
$$

where $u \in R^{m}, y \in R^{m}, \quad x \in R^{n \geq m}$, and $C \in R^{m \times n}$ is a constant full rank matrix. This system can be brought to discrete time form with a sampling period $\mathrm{T}$ :

$$
\begin{aligned}
& x_{k+1}=f\left(x_{k}, u_{k}\right) \\
& y_{k+1}=C x_{k+1}+d_{k}
\end{aligned}
$$

where $\frac{\partial^{2} f\left(x_{k}, u_{k}\right)}{\partial u_{k}^{2}} \rightarrow 0$ as $T \rightarrow 0$, due to the linearity of (2.1) with respect to $u$. In words, (2.3) is arbitrarily linear in $u_{k}$ for a fixed $x_{k}$.

Let subscripts denote time step index and superscripts denote learning trial index. The method of Arimoto et al [1] takes the form:

$$
u_{k}^{i+1}-u_{k}^{i}=-\left(\hat{J}_{k}^{i}\right)^{-1} y_{k+1}^{i}
$$

where

$$
\hat{J}_{k}^{i}=C \frac{\partial f\left(x_{k}, u_{k}\right)}{\partial u_{k}}
$$

Since the system model (2.3)/(2.4) is not perfect,

$$
\hat{J}_{k}^{i} \neq J_{k}^{i}=\frac{\partial y_{k+1}^{i}}{\partial u_{k}^{i}}
$$

Under the condition

$$
\left\|I-J \hat{J}^{-1}\right\|<1
$$

the errors $\left\|y_{2}^{i}\right\| \rightarrow 0$ monotonically for the sequence $u_{1}^{i}$ for small enough $T$. Under the condition in Avrachenkov [2]:

$$
\left\|I-\hat{J}^{-1} J\right\|<1
$$

instead $\left\|u_{1}^{i}-u_{1}^{*}\right\| \rightarrow 0$ monotonically where $u_{1}^{*}$ is the $u_{1}$ that makes $y_{2}=0$.

Now consider the case where the inputs are bounded:

$$
u_{\min }(i) \leq u(i) \leq u_{\max }(i),(\mathrm{i}=1, \ldots, \mathrm{m}) \quad(2.10)
$$

and where there exists a $u_{k},(\mathrm{k}=1, \ldots, \mathrm{N})$, where $\mathrm{N}$ is the number of sampling periods, that satisfies (2.3), (2.4), and $y_{k}=0 \forall k$. Under this scenario, we propose the following simple learning control update rule:

$$
u_{k}^{i+1}=\operatorname{box}\left(u_{k}^{i}-\left(\hat{J}_{k}^{i}\right)^{-1} y_{k+1}^{i}\right)
$$

where $u=b o x(\hat{u})$ means

$$
u(i)=\left\{\begin{array}{c}
\hat{u}(i) \text { if } u_{\min }(i) \leq \hat{u}(i) \leq u_{\max }(i) \\
u_{\max }(i) \text { if } \hat{u}(i)>u_{\max }(i) \\
u_{\min }(i) \text { if } \hat{u}(i)<u_{\min }(i)
\end{array}\right\},(\mathrm{i}=1, \ldots, \mathrm{m})
$$

The rationale for this update rule is contained in Theorem 2.1 below and its proof.

Theorem 2.1: Suppose $u^{*} \in R^{m}$ is a point satisfying $u_{\min }(i) \leq u^{*}(i) \leq u_{\max }(i),(\mathrm{i}=1, \ldots, \mathrm{m})$. Let $\hat{u}$ be an arbitrary point in $R^{m}$. Let $u=b o x(\hat{u})$. Then,

$$
\left\|u-u^{*}\right\| \leq\left\|\hat{u}-u^{*}\right\|
$$

Proof of Theorem 2.1: By the mere construction of $u$ from $\hat{u}$ and the fact that $u_{\text {min }}(i) \leq u^{*}(i) \leq u_{\max }(i)$, we have $\left|u(i)-u^{*}(i)\right| \leq\left|\hat{u}(i)-u^{*}(i)\right|, \quad \forall i$. Thus, $\left\|u-u^{*}\right\| \leq\left\|\hat{u}-u^{*}\right\|$ follows immediately. QED.

Thus we see that conditions for monotonically decreasing distance to the solution, in particular those in Avrachenkov [2], are sufficient for the chopping update rule (2.11) to converge to the solution. However, as stated below in Remark 2.1, the standard condition (2.8) for monotonically decreasing output error is not sufficient to guarantee convergence of the chopping update rule (2.11).

Remark 2.1: The input-chopping learning control update rule (2.11) can fail to converge to the solution under the standard condition (2.8), even for a linear dynamic system. We can prove this with a small example. Consider the following linear three-input/three-output system:

$$
y=J u-b
$$

where

$$
\begin{aligned}
& J=\left[\begin{array}{ccc}
0.6560 & -0.9137 & -1.1544 \\
-1.9967 & -0.6481 & 1.7580 \\
-0.3209 & -1.3331 & -1.3797
\end{array}\right] \\
& \hat{J}=\left[\begin{array}{ccc}
1.1180 & -0.5024 & -1.2048 \\
-1.5582 & -0.2416 & 1.9289 \\
-0.6724 & -1.7287 & -1.2587
\end{array}\right] \\
& b=[-0.4912,-0.3186,-1.2961]^{T}
\end{aligned}
$$

and initial input

$$
u^{\prime}=[-0.5905,0.2341,-0.5758]^{T}
$$

with bounds $-u_{\max } \leq u \leq u_{\max }$ :

$$
u_{\max }=[0.5905,0.2341,0.5758]^{T}
$$


The pair $(J, \hat{J})$ satisfies (2.8), but does not satisfy (2.9). Application of the rule (2.11) leads to no change in the input $u$ even though the input $u^{\prime}$ does not make $y=0$. That is, the chopping update rule gets stuck even though the standard Jacobian error condition (2.8) is satisfied.

\section{Conclusion}

Under the Jacobian error condition presented in Avrachenkov [2] and a simple chopping rule for ensuring learning iterates are within their limits, the result is a very simple extension of the iterative learning control algorithm of Arimoto et al [1] to the case of bounded inputs. The key to the development is the Jacobian error condition associated with monotonicity of the input error norm, rather than the usual condition for monotonicity of the output error norm. The chopping rule does not affect the monotonicity of input error norm, while it can in general affect the monotonicity of the output error. More specifically, the former Jacobian error condition is sufficient for convergence of the iterative learning control algorithm proposed herein while the latter ('standard') Jacobian error condition is not.

\section{References}

[1] Arimoto, S., et al, "Bettering Operation of Robots by Learning," Journal of Robotic Systems, Vol. 1, No. 2, 1984, pp. 123-140.

[2] Avrachenkov, K., "Iterative Learning Control Based On Quasi-Newton Methods," 1998 Conference on Decision and Control, pp. 170-174.

[3] Bazaraa, M., et al, Nonlinear Programming, Theory and Applications, Second Edition, New York: John Wiley and Sons, Inc., 1979.

[4] Cheah, C. and Wang, D., "Learning Control for a Class of Nonlinear Differential-Algebraic Systems With Application to Constrained Robots," Journal of Robotic Systems, Vol. 13, No. 3, 1996, pp. 141-151.

[5] Chen, C. and Peng, S., "Learning Control of Process Systems with Hard Input Constraints," Journal of Process Control, Vol. 9, 1999, pp. 151-160.

[6] Driessen, B., Sadegh, N., and Kwok, K., "A Robust Line Search for Learning Control," Conference on Decision and Control, 1998.

[7] Gorinevsky, D., "An Algorithm for On-Line Parametric Nonlinear Least Square Optimization," 33rd IEEE CDC, Lake Buena Vista, Florida, December, 1994.

[8] Gorinevsky, D., "An Application of On-Line Parametric Optimization to Task-Level Learning Control," American Control Conference, Seattle, Washington, June 1995, pp. 862-866.

[9] Gorinevsky, G. et al, "Learning Approximation of Feedforward Dependence on the Task Parameters:
Experiments in Direct-Drive Manipulator Tracking," American Control Conference, Seattle Washington, 1995, pp. 883-887.

[10] Horowitz, Roberto, "Learning Control of Robot Manipulators," ASME Journal of Dynamic Systems, Measurement, and Control, Vol. 115, June 1993, pp. 402-411.

[11]Moore, K.L., 1993, Iterative Learning Control for Deterministic Systems, Advances in Industrial Control Series (London, U.K.: Springer-Verlag).

[12] Sadegh, N. and Driessen, B., "Minimum Time Trajectory Learning," ASME Joumal of Dynamic Systems, Measurement and Control, Vol. 121,.June 1999, pp. 213-217. 